\title{
Design of Multi-Directional Multi-Scroll Chaotic Attractors Based on Fractional Differential Systems
}

\author{
Weihua Deng \\ School of Mathematics and Statistics \\ Lanzhou University \\ Lanzhou 730000, P. R. China
}

\author{
Jinhu Lü \\ Key Laboratory of Systems and Control, Institute \\ of Systems Science, Academy of Mathematics and \\ Systems Science, Chinese Academy of Sciences \\ Beijing 100080, P. R. China \\ Email: jhlu@iss.ac.cn
}

\begin{abstract}
A novel approach is proposed for generating multidirectional multi-scroll chaotic attractors from the fractional differential systems, including one-directional (1-D) $n$-scroll, two-directional (2-D) $n \times m$-grid scroll, and three-directional (3D) $n \times m \times l$-grid scroll chaotic attractors. It is the first time in the literature to report the multi-directional multi-scroll chaotic attractors from a fractional differential systems. Furthermore, some underlying dynamical mechanics are briefly investigated for the fractional differential multi-scroll systems.
\end{abstract}

\section{INTRODUCTION}

Over the past few decades, fractional differential systems have received an increasing attention from many researchers. On the one hand, more and more fractional differential systems have been applied to the interdisciplinary fields, including model acoustics and thermal systems, rheology and modeling of materials and mechanical systems, signal processing and systems identification, control and robotics, etc. [1-2]. On the other hand, many real-world systems modeled by fractional calculus also display rich fractional dynamical behaviors, such as viscoelastic systems, colored noise, boundary layer effects in ducts, and electrode- electrolyte polarization [1-2].

Yalcin and his colleagues [3-4] introduced various methods for generating multi-scroll chaotic attractors. Lü and his colleagues proposed the hysteresis series and saturated series methods for generating one-directional (1-D) $n$-scroll, twodirectional (2-D) $n \times m$-grid scroll, and three-directional (3-D) $n \times m \times l$-grid scroll chaotic attractors with rigorously mathematical proofs and experimental verifications [58]. Recently, Lü and Chen [8] reviewed the main advances in theories, approaches, and applications of multi-scroll chaos generation over the last two decades. Compared with the single-scroll chaotic attractors, the multi-scroll chaotic attractors have much higher complexity and more adjustability, which makes multi-scroll chaotic attractors have a generally applied prospect in various chaos-based information technologies, such as encryption and secure communication $[4,8]$.

Many researchers surprisingly found that many nonlinear fractional differential systems also display complex dynamical behaviors. Recently, Ahmad [9] introduced a step function method for creating $n$-scroll chaotic attractors from fractional order systems. However, the design of multi-directional multiscroll chaotic attractors is also a very challenging question.
Therefore, it is very interesting to ask whether the fractional differential systems can also generate multi-directional multiscroll chaotic attractors as the classical differential systems. This paper will give a positive answer to this question.

This paper proposes a systematic design approach for creating multi-directional multi-scroll chaotic attractors from a fractional differential system via saturated function series controller, including 1-D $n$-scroll, 2-D $n \times m$-grid scroll, and 3-D $n \times m \times l$-grid scroll chaotic attractors. It should be especially pointed out that the fractional differential multiscroll systems are quite different from the classical differential multi-scroll systems because the fractional derivative is a nonlocal operator [10].

The rest of this paper is organized as follows. In Section II, some preliminaries are introduced for the fractional differential systems. Then the saturated function series approach is proposed for generating multi-directional multi-scroll chaotic attractors in Section III. And the conclusions are finally drawn in Section IV.

\section{Fractional DifFEREnTIAL SyStemS}

This section briefly introduces some background knowledge for fractional differential systems.

As we know now, there are several different definitions for the fractional differential operator. Hereafter, the fractional differential operator is described by

$$
D_{*}^{\alpha} y(x)=J^{m-\alpha} y^{(m)}(x), \quad \alpha>0,
$$

where $m=\lceil\alpha\rceil$, i.e., $m$ is the first integer which is not less than $\alpha, y^{(m)}$ is the general $m$-order derivative, and $J^{\beta}$ is the $\beta$-order Riemann-Liouville integral operator which is given by

$$
J^{\beta} z(x)=\frac{1}{\Gamma(\beta)} \int_{0}^{x}(x-t)^{\beta-1} z(t) d(t), \quad \beta>0 .
$$

Here, the operator $D_{*}^{\alpha}$ is called " $\alpha$-order Caputo differential operator" which is widely used in engineering field.

\section{A. Preliminaries}

It is well known that $D_{*}^{\alpha} c=0$ for any constant $c$ and $\alpha \in \mathbb{R}^{+}$. In the following, one introduces a basic definition for the fractional differential systems. 
Definition 1: The roots of the equation $f(X)=0$ are called the equilibria of fractional differential system $D_{*}^{\alpha} X=$ $f(X)$, where $X=\left(x_{1}, x_{2}, \cdots, x_{n}\right)^{T} \in \mathbb{R}^{n}, f(X) \in R^{n}$ and $D_{*}^{\alpha} X=\left(D_{*}^{\alpha_{1}} x_{1}, D_{*}^{\alpha_{2}} x_{2}, \cdots, D_{*}^{\alpha_{n}} x_{n}\right)^{T}, \alpha_{i} \in \mathbb{R}^{+}, i=$ $1,2, \cdots, n$.

Lemma 1: [10] The equilibrium $X_{0}=-\mathbf{A}^{-1} \mathbf{b}$ of the fractional linear autonomous system $D_{*}^{\alpha} X=\mathbf{A} X+\mathbf{b}$ is asymptotically stable if and only if $\left|\arg \left(\lambda_{i}\right)\right|>\alpha_{0} \pi / 2$ for $i=1,2, \cdots, n$, where $\lambda_{i}$ are the eigenvalues of matrix $\mathbf{A}, X=\left(x_{1}, x_{2}, \cdots, x_{n}\right)^{T} \in \mathbb{R}^{n}, \mathbf{A} \in \mathbb{R}^{n} \times \mathbb{R}^{n},|\mathbf{A}| \neq$ $0, \mathbf{b} \in \mathbb{R}^{n}$, and $D_{*}^{\alpha} X=\left(D_{*}^{\alpha_{1}} x_{1}, D_{*}^{\alpha_{2}} x_{2}, \cdots, D_{*}^{\alpha_{n}} x_{n}\right)^{T}$, $\alpha_{1}=\alpha_{2}=\cdots=\alpha_{n}=\alpha_{0} \in \mathbb{R}^{+}, i=1,2, \cdots, n$.

The simplest saturated function is described by

$$
f_{0}(x ; k)= \begin{cases}k, & \text { if } x>1 \\ k x, & \text { if }|x| \leq 1 \\ -k, & \text { if } x<-1\end{cases}
$$

where $k>0$ is the slope of the middle segment. The upper radial $\left\{f_{0}(x ; k)=k \mid x \geq 1\right\}$ and lower radial $\left\{f_{0}(x ; k)=-k \mid x \leq-1\right\}$ are called saturated plateaus, and the segment $\left\{f_{0}(x ; k)=k x|| x \mid \leq 1\right\}$ between the two saturated plateaus is called the saturated slope.

Definition 2: [8] The piecewise linear (PWL) function

$$
f(x ; k, h, p, q)=\sum_{i=-p}^{q} f_{i}(x ; k, h)
$$

is called a saturated function series, where $k>0$ and $h>2$ are the slope and saturated delay time of the saturated function series (2), respectively, $p$ and $q$ are positive integers, and

$$
f_{i}(x ; k, h)= \begin{cases}2 k, & \text { if } x>i h+1 \\ k(x-i h)+k, & \text { if }|x-i h| \leq 1 \\ 0, & \text { if } x<i h-1\end{cases}
$$

and

$$
f_{-i}(x ; k, h)= \begin{cases}0, & \text { if } x>-i h+1 \\ k(x+i h)-k, & \text { if }|x+i h| \leq 1 \\ -2 k, & \text { if } x<-i h-1\end{cases}
$$

\section{B. The Basic Fractional Linear Autonomous System}

This subsection introduces a basic fractional linear autonomous system and further discuss its potential ability to generate the multi-directional multi-scroll chaotic attractors via a suitable controller.

The fundamental fractional linear autonomous system is given by

$$
\left(\begin{array}{c}
D_{*}^{\alpha_{1}} x \\
D_{*}^{\alpha_{2}} y \\
D_{*}^{\alpha_{3}} z
\end{array}\right)=\left(\begin{array}{ccc}
0 & 1 & 0 \\
0 & 0 & 1 \\
-a & -b & -c
\end{array}\right)=\left(\begin{array}{l}
x \\
y \\
z
\end{array}\right) \equiv \mathbf{A}\left(\begin{array}{l}
x \\
y \\
z
\end{array}\right)
$$

where $x, y, z$ are state variables, $a, b, c$ are positive real constants, and $\alpha_{i} \in(0,1), i=1,2,3$.

When $\alpha_{i} \in(0,1)$ for $i=1,2,3$ are rational numbers, system (3) can be transformed into its equivalent system with the same fractional orders [10]. Therefore, one only need to discuss the fractional differential system

$$
D_{*}^{\alpha} X=\mathbf{A} X,
$$

where $X=(x, y, z)^{T}, D_{*}^{\alpha} X=\left(D_{*}^{\alpha_{1}} x, D_{*}^{\alpha_{2}} y, D_{*}^{\alpha_{3}} z\right)^{T}$, and $\alpha_{1}=\alpha_{2}=\alpha_{3}=\alpha_{0} \in(0,1)$.

The characteristic equation of the coefficient matrix $\mathbf{A}$ of system (4) is described by

$$
\lambda^{3}+c \lambda^{2}+b \lambda+a=0 .
$$

Note that (5) is not the characteristic equation of (4) because the characteristic equation of fractional differential system has different meaning [10].

From Lemma 1 , the stability of the equilibrium $(0,0,0)$ of fractional differential system (4) is completely determined by the eigenvalues of (5). Denote $\hat{q}=(2 / 27) c^{3}-(1 / 3) b c+a$, and $\Delta=\left(a c^{3} / 27\right)-\left(b^{2} c^{2} / 108\right)-(a b c / 6)+\left(b^{3} / 27\right)+\left(a^{2} / 4\right)$. From (5), one has

$$
\lambda_{1}=-\frac{c}{3}+\sqrt[3]{-\frac{\hat{q}}{2}+\sqrt{\Delta}}+\sqrt[3]{-\frac{\hat{q}}{2}-\sqrt{\Delta}}
$$

and

$$
\begin{aligned}
\lambda_{2,3}= & -\frac{c}{3}-\frac{1}{2}\left(\sqrt[3]{-\frac{\hat{q}}{2}+\sqrt{\Delta}}+\sqrt[3]{-\frac{\hat{q}}{2}-\sqrt{\Delta}}\right) \\
& \pm \frac{\sqrt{3}}{2} i\left(\sqrt[3]{-\frac{\hat{q}}{2}+\sqrt{\Delta}}-\sqrt[3]{-\frac{\hat{q}}{2}-\sqrt{\Delta}}\right) \\
= & \beta \pm \gamma i .
\end{aligned}
$$

Our theoretical and numerical analysis show that system (4) with a saturated function series controller (2) may generate chaotic behavior under the condition of $\lambda_{1}<0, \beta>0$, $\gamma \neq 0$ and $|\arctan (\gamma / \beta)|<\alpha_{0} \pi / 2$. Here $(0,0,0)$ is a saddle point of index 2 of (4).

Hereafter, one always supposes that

$$
\begin{aligned}
& \Delta=\frac{a c^{3}}{27}-\frac{b^{2} c^{2}}{108}-\frac{a b c}{6}+\frac{b^{3}}{27}+\frac{a^{2}}{4}>0, \\
& \lambda_{1}=-\frac{c}{3}+\sqrt[3]{-\frac{\hat{q}}{2}+\sqrt{\Delta}}+\sqrt[3]{-\frac{\hat{q}}{2}-\sqrt{\Delta}}<0, \\
& \beta=-\frac{c}{3}-\frac{1}{2}\left(\sqrt[3]{-\frac{\hat{q}}{2}+\sqrt{\Delta}}+\sqrt[3]{-\frac{\hat{q}}{2}-\sqrt{\Delta}}\right)>0, \\
& |\arctan (\gamma / \beta)|<\alpha_{0} \pi / 2 .
\end{aligned}
$$

\section{The Numerical Computational Schemes}

In the following, the predictor-corrector scheme is used to numerically solve the fractional differential equation. Noticed that this scheme is a natural generalization of the known Adams-Bashforth-Moulton scheme [10-12].

The fractional differential equation is given by

$$
\left\{\begin{array}{l}
D_{*}^{\alpha_{1}} x(t)=l_{1}(x, y, z) \\
D_{*}^{\alpha_{2}} y(t)=l_{2}(x, y, z) \\
D_{*}^{\alpha_{3}} z(t)=l_{3}(x, y, z)
\end{array}\right.
$$

where the initial values $x(0)=x_{0}, y(0)=y_{0}, z(0)=z_{0}$, $\alpha_{i} \in(0,1), i=1,2,3$, and $0 \leq t \leq T$. Thus it is equivalent to the Volterra integral equation

$$
\left\{\begin{array}{l}
x(t)=x(0)+\frac{1}{\Gamma\left(\alpha_{1}\right)} \int_{0}^{t}(t-s)^{\alpha_{1}-1} l_{1}(x(s), y(s), z(s)) d s \\
y(t)=y(0)+\frac{1}{\Gamma\left(\alpha_{2}\right)} \int_{0}^{t}(t-s)^{\alpha_{2}-1} l_{2}(x(s), y(s), z(s)) d s \\
z(t)=z(0)+\frac{1}{\Gamma\left(\alpha_{3}\right)} \int_{0}^{t}(t-s)^{\alpha_{3}-1} l_{3}(x(s), y(s), z(s)) d s .
\end{array}\right.
$$


Let $h=\frac{T}{N}, t_{n}=n h, n=0,1, \cdots, N \in Z^{+}$. Discretizing the Volterra integral equation as above yields

$$
\left\{\begin{aligned}
x_{h}\left(t_{n+1}\right)= & x(0)+\frac{h^{\alpha_{1}}}{\Gamma\left(\alpha_{1}+2\right)} \times\left(x_{h}^{p}\left(t^{n+1}\right)+\left(2^{\left(\alpha_{1}+1\right)}\right.\right. \\
& \left.-2) \times l_{1}(x(s), y(s), z(s))+\text { temp } 1\right) \\
y_{h}\left(t_{n+1}\right)= & y(0)+\frac{h^{\alpha_{2}}}{\Gamma\left(\alpha_{2}+2\right)} \times\left(y_{h}^{p}\left(t^{n+1}\right)+\left(2^{\left(\alpha_{2}+1\right)}\right.\right. \\
& \left.-2) \times l_{2}(x(s), y(s), z(s))+\text { temp2 }\right) \\
z_{h}\left(t_{n+1}\right)= & z(0)+\frac{h^{\alpha_{3}}}{\Gamma\left(\alpha_{3}+2\right)} \times\left(z_{h}^{p}\left(t^{n+1}\right)+\left(2^{\left(\alpha_{3}+1\right)}\right.\right. \\
& \left.-2) \times l_{3}(x(s), y(s), z(s))+\text { temp3 }\right),
\end{aligned}\right.
$$

where

$$
\begin{aligned}
& \left\{\begin{aligned}
x_{h}^{p}\left(t_{n+1}\right)= & x(0)+\frac{h^{\alpha_{1}}}{\Gamma\left(\alpha_{1}+2\right)} \times\left(\left(2^{\left(\alpha_{1}+1\right)}-1\right)\right. \\
& \left.\times l_{1}(x(s), y(s), z(s))+\text { temp } 1\right) \\
y_{h}^{p}\left(t_{n+1}\right)= & y(0)+\frac{h^{\alpha_{2}}}{\Gamma\left(\alpha_{2}+2\right)} \times\left(\left(2^{\left(\alpha_{2}+1\right)}-1\right)\right. \\
& \left.\times l_{2}(x(s), y(s), z(s))+\text { temp2 }\right) \\
z_{h}^{p}\left(t_{n+1}\right)= & z(0)+\frac{h^{\alpha}}{\Gamma\left(\alpha_{3}+2\right)} \times\left(\left(2^{\left(\alpha_{3}+1\right)}-1\right)\right. \\
& \left.\times l_{3}(x(s), y(s), z(s))+\text { temp3 }\right)
\end{aligned}\right. \\
& \left\{\begin{array}{l}
\text { temp1 }=\frac{h^{\alpha_{1}}}{\Gamma\left(\alpha_{1}+2\right)} \sum_{j=0}^{n-1} a_{1, j, n+1} l_{1}\left(x_{h}\left(t_{j}\right), y_{h}\left(t_{j}\right), z_{h}\left(t_{j}\right)\right) \\
\text { temp2 }=\frac{h^{\alpha_{2}}}{\Gamma\left(\alpha_{2}+2\right)} \sum_{j=0}^{n-1} a_{2, j, n+1} l_{2}\left(x_{h}\left(t_{j}\right), y_{h}\left(t_{j}\right), z_{h}\left(t_{j}\right)\right) \\
\text { temp3 }=\frac{h^{\alpha_{3}}}{\Gamma\left(\alpha_{3}+2\right)} \sum_{j=0}^{n-1} a_{3, j, n+1} l_{3}\left(x_{h}\left(t_{j}\right), y_{h}\left(t_{j}\right), z_{h}\left(t_{j}\right)\right),
\end{array}\right. \\
& = \begin{cases}a_{i, j, n+1} & n^{\alpha_{i}+1}-\left(n-\alpha_{i}\right)(n+1)^{\alpha_{i}}, \\
(n-j+2)^{\alpha_{i}+1}+(n-j)^{\alpha_{i}+1} & 1 \leq j \leq n-1 \\
-2(n-j+1)^{\alpha_{i}+1}, & \end{cases}
\end{aligned}
$$

where $i=1,2,3$.

Moreover, the error estimate is described by

$$
\begin{gathered}
\max \left\{\max _{0 \leq j \leq N}\left|x\left(t_{j}\right)-x_{h}\left(t_{j}\right)\right|, \max _{0 \leq j \leq N}\left|y\left(t_{j}\right)-y_{h}\left(t_{j}\right)\right|,\right. \\
\left.\max _{0 \leq j \leq N}\left|z\left(t_{j}\right)-z_{h}\left(t_{j}\right)\right|\right\}=O\left(h^{q}\right),
\end{gathered}
$$

where $q=\min \left\{1+\alpha_{1}, 1+\alpha_{2}, 1+\alpha_{3}\right\}$.

\section{DESIGN OF MULTI-DIRECTIONAL MULTI-SCROLL CHAOTIC ATTRACTORS}

In this section, a systematic control approach is introduced for creating multi-directional multi-scroll chaotic attractors from the fundamental fractional differential linear system (3) via saturated function series controller (2). It includes 1-D $n$-scroll, 2-D $n \times m$-grid scroll, and 3-D $n \times m \times l$-grid scroll chaotic attractors. The controlled fractional differential system is described by

$$
D_{*}^{\alpha} X=\mathbf{A} X+\mathbf{B} U(X),
$$

where

$$
\mathbf{B}=\left(\begin{array}{ccc}
0 & -\frac{d_{2}}{b} & 0 \\
0 & 0 & -\frac{d_{3}}{c} \\
d_{1} & d_{2} & d_{3}
\end{array}\right),
$$

$X=(x, y, z)^{T}, D_{*}^{\alpha} X=\left(D_{*}^{\alpha_{1}} x, D_{*}^{\alpha_{2}} y, D_{*}^{\alpha_{3}} z\right)^{T}$, and $U(X)$ is the saturated functions series controller, which is designed as follows: i) 1-D $n-$ scroll:

$$
\mathbf{U}(\mathbf{X})=\left(\begin{array}{c}
f\left(x ; k_{1}, h_{1}, p_{1}, q_{1}\right) \\
0 \\
0
\end{array}\right),
$$

ii) 2-D $n \times m$-grid scroll:

$$
U(X)=\left(\begin{array}{c}
f\left(x ; k_{1}, h_{1}, p_{1}, q_{1}\right) \\
f\left(y ; k_{2}, h_{2}, p_{2}, q_{2}\right) \\
0
\end{array}\right),
$$

iii) 3-D $n \times m \times l$-grid scroll:

$$
U(X)=\left(\begin{array}{c}
f\left(x ; k_{1}, h_{1}, p_{1}, q_{1}\right) \\
f\left(y ; k_{2}, h_{2}, p_{2}, q_{2}\right) \\
f\left(z ; k_{3}, h_{3}, p_{3}, q_{3}\right)
\end{array}\right),
$$

where $f\left(x ; k_{1}, h_{1}, p_{1}, q_{1}\right), f\left(y ; k_{2}, h_{2}, p_{2}, q_{2}\right), f\left(z ; k_{3}, h_{3}\right.$ , $\left.p_{3}, q_{3}\right)$ are defined by (2), and $a, b, c, d_{1}, d_{2}, d_{3}$ are positive constants.

Fig. 1 (a) shows a 1-D 6-scroll chaotic attractor with fractional order $(0.85,0.9,0.9)$ and Lyapunov exponents spectrum $L E_{1}=0.2251, L E_{2}=0, L E_{3}=-1.2143$, where the system parameters are given by $a=2, b=1, c=$ $0.6, d_{1}=2, k_{1}=10$. Fig. 1 (b) displays a 2 -D $6 \times 6$-grid scroll chaotic attractor with fractional order $(0.8,0.9,1.0)$ and Lyapunov exponents spectrum $L E_{1}=0.2131, L E_{2}=$ $0, L E_{3}=-1.5718$, where the system parameters are given by $a=2, b=1, c=0.5, d_{1}=2, d_{2}=1, k_{1}=50, k_{2}=50$. Fig. 2 shows a 3-D $6 \times 6 \times 6$-grid scroll chaotic attractor with fractional order $(0.9,0.9,0.9)$ and Lyapunov exponents spectrum $L E_{1}=0.3629, L E_{2}=0, L E_{3}=-1.2742$, where the system parameters are given by $a=2.2, b=1.3$, $c=0.6, d_{1}=2.2, d_{2}=1.3, d_{3}=0.6, k_{1}=100, k_{2}=40$, and $k_{3}=40$.

Parameters $p_{1}, q_{1}$ control the numbers of scrolls in negative and positive $x$-directions, respectively. Parameters $p_{2}, q_{2}$ control the numbers of scrolls in negative and positive $y$-directions, respectively. Parameters $p_{3}, q_{3}$ control the numbers of scrolls in negative and positive $z$-directions, respectively.

\section{CONCLUSIONS}

This paper has introduced a systematic approach for creating multi-directional multi-scroll chaotic attractors from the fractional differential systems. It includes the 1-D $n$-scroll, 2-D $n \times m$-grid scroll, and 3-D $n \times m \times l$-grid scroll attractors. In particular, it is the first time in the literature to report the multi-directional multi-scroll chaotic attractors from a fractional differential systems. Moreover, some underlying dynamical mechanics are further studied for the generation of multi-directional multi-scroll chaotic attractors in the fractional differential systems. We also find that each saturated plateau corresponds to a unique equilibrium and also corresponds to the unique scroll of the whole attractor. Last but not least, one can arbitrarily design a multi-directional multiscroll chaotic attractor with the desired number of scrolls, the desired spatial positions and orientations by adjusting the 

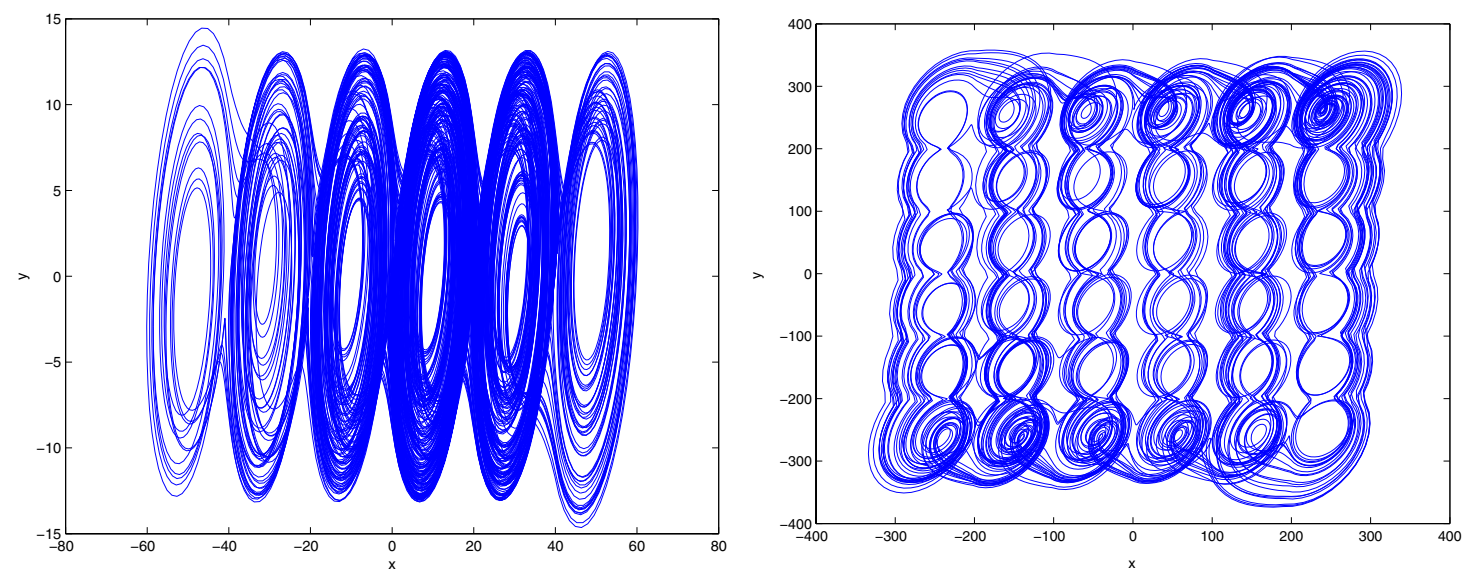

Fig. 1. 1-D $n$-scroll and 2-D 2-D $n \times m$-grid scroll attractors. (a) 6 -scroll with fractional order $(0.85,0.9,0.9)$. (b) $6 \times 6$-grid scroll with fractional order $(0.8,0.9,1.0)$.
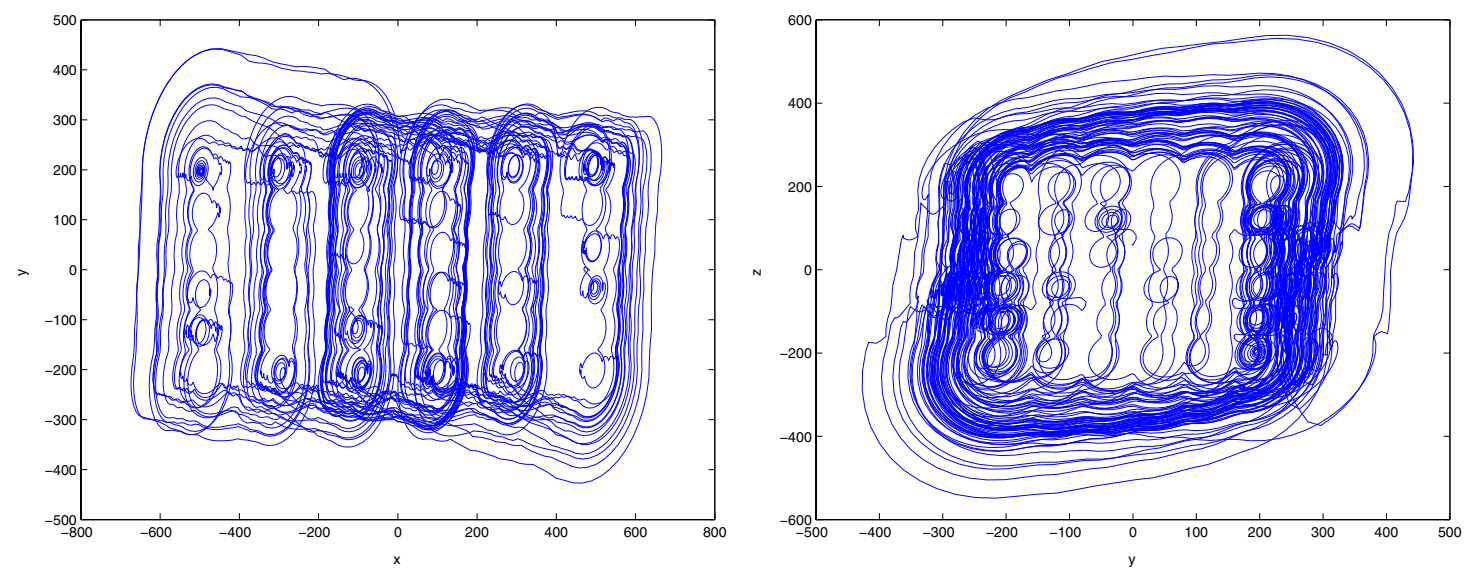

Fig. 2. A 3-D $6 \times 6 \times 6$-grid scroll attractor with fractional order $(0.9,0.9,0.9)$. (a) $x-y$ plane. (b) $y-z$ plane.

system parameters $p_{i}$ and $q_{i}(i=1,2,3)$ of the saturated function series controller.

\section{ACKNOWLEDGMENT}

This work was supported by the National Natural Science Foundation of China under Grants 60304017, 20336040, and 60221301, the Scientific Research Startup Special Foundation on Excellent PhD Thesis and Presidential Award of Chinese Academy of Sciences.

\section{REFERENCES}

[1] R. C. Koeller, "Application of fractional calculus to the theory of viscoelasticity," J. Appl. Mech., vol. 51, pp. 229-307, 1984.

[2] D. Kusnezov, A. Bulgac, and G. D. Dang, "Quantum levy processes and fractional kinetics," Phys. Rev. Lett., vol. 82, pp. 1136-1139, 1999.

[3] J. A. K. Suykens and J. Vandewalle, "Generation of $n$-double scrolls ( $n=1,2,3,4, \cdots)$," IEEE Trans. Circuits Syst. I, vol. 40, no. 11, pp. 861-867, Nov. 1993.

[4] M. E. Yalcin, J. A. K. Suykens, and J. Vandewalle, Cellular Neural Networks, Multi-Scroll Chaos and Synchronization. Singapore: World Scientific, 2005.
[5] J. Lü, X. Yu, and G. Chen, "Generating chaotic attractors with multiple merged basions of attraction: A switching piecewise-linear control approach," IEEE Trans. Circuits Syst. I, vol. 50, no. 2, pp. 198-207, Feb. 2003.

[6] J. Lü, F. Han, X. Yu, and G. Chen, "Generating 3-D multi-scroll chaotic attractors: A hysteresis series switching method," Automatica, vol. 40, no. 10, pp. 1677- 1687, Oct. 2004.

[7] J. Lü, S. M. Yu, H. Leung, and G. Chen, "Experimental verification of multi-directional multi-scroll chaotic attractors," IEEE Trans. Circuits Syst. I, vol. 53, no. 1, pp. 149-165, Jan. 2006.

[8] J. Lü and G. Chen, "Multi-scroll chaos generation: Theories, methods and applications," Int. J. Bifurcation and Chaos, vol. 16, no. 4, pp. 775858, Apr. 2006.

[9] W. Ahmad, "Generation and control of multi-scroll chaotic attractors in fractional order systems," Chaos, Solitons \& Fractals, vol. 25, no. 3, 727-735, 2005.

[10] W. H. Deng, Y. J. Wu, and C. P. Li, "Stability analysis of differential equations with time-dependent delay," Int. J. Bifurcation and Chaos, vol. 16, no. 2, pp. 465-472, Feb. 2006.

[11] K. Diethelm, N. J. Ford, and A. D. Freed, "A predictor-corrector approach for the numerical solution of fractional differential equations," Nonl. Dyn., vol. 29, pp. 3-22, 2002.

[12] K. Diethelm, N. J. Ford, and A. D. Freed, "Detailed error analysis for a fractional Adams method," Numerical algorithms, vol. 36, pp. 31-52, 2004. 